\title{
Type A aortic dissection: Has surgical outcome improved with time?
}

Pradeep Narayan, FRCS, Chris A. Rogers, PhD, Ian Davies, FRCA, Gianni D. Angelini, FRCS, and Alan J. Bryan, FRCS (CTh)

Objective: The study objective was to determine whether developments in surgical, anesthetic, and perfusion techniques in the treatment of type A aortic dissection have resulted in improved clinical outcome.

Methods: A consecutive series of 165 patients undergoing surgical repair of type A aortic dissection performed between April of 1992 and March of 2006 in a single center were analyzed. Operations were grouped in 2 time frames of equal length (before April of 1999 vs from April of 1999 onward).

Results: There were 30 in-hospital deaths $(18.2 \%)$, and the death rate was similar in the 2 time periods. Patients who underwent operation in the recent era compared with the earlier era were older (median 62 years [interquartile range 51-68] vs 59 years [45-68], $P=.18$ ), with a significantly higher incidence of concomitant coronary artery disease (13 [18\%] vs $5[7 \%], P=.03])$ and significantly worse (moderate to poor) left ventricular function (33 $[40 \%]$ vs $13[18 \%], P=.002$ ). The duration of circulatory arrest was shorter in the recent era (median 31 minutes [interquartile range 26.5-39] vs 37.5 minutes [31-45], $P=.009$ ), with a higher incidence of concomitant procedures $(19[21 \%]$ vs 10 [14\%], $P=.22)$. Except for total hospital stay, which increased over time, there were no significant differences in postoperative outcome.

Conclusion: Despite the adoption of techniques to improve outcome for patients with type A dissection, mortality remains unchanged. A deteriorating risk profile and factors relating to the disease process itself may explain this observation.

Acute type A aortic dissection carries a high early mortality with an exponential decline in survival with time. The incidence of aortic dissection worldwide is estimated to be 0.5 to 2.95 per 100,000 per year. ${ }^{1}$ Without any intervention it is associated with a $90 \%$ mortality within 2 weeks. ${ }^{2}$ The hospital mortality with surgical treatment remains high and in contemporary clinical series is between $5 \%$ and $25 \% .^{2-4}$

Techniques in this area have evolved, and a number of groups from major cardiac surgical institutions around the world have shown that outcomes for surgically treated patients with type A dissection improved with time. ${ }^{5,6}$

However, more contemporary series have failed to show a similar trend. ${ }^{3,7}$ It is perhaps noteworthy that some of the best published results have been achieved by the application of some of the simplest surgical principles in this condition. ${ }^{8,9}$

Why then might we hypothesize that surgical outcomes should have improved since the early 1990s? The improved diagnostic capabilities in local hospitals with around-theclock availability of high-quality computed tomography (CT) scanning has enabled quicker and more accurate diagnosis and earlier referral to the specialist center. Because we know outcome is directly related to the preoperative state, ${ }^{10,11}$ this should favor improved results. Isolated replacement of the ascending aorta remains the mainstay of

From the Bristol Heart Institute, Bristol Royal Infirmary, Bristol, United Kingdom. Received for publication Dec 5, 2007; revisions received April 7, 2008; accepted for publication May 4, 2008.

Address for reprints: Alan J. Bryan, FRCS (CTh), Consultant Cardiac Surgeon, Bristol Heart Institute, Bristol Royal Infirmary, Bristol, BS2 8HW, United Kingdom

(E-mail: Alan.Bryan@ubht.nhs.uk).

J Thorac Cardiovasc Surg 2008;136:1172-7

$0022-5223 / \$ 34.00$

Copyright (c) 2008 by The American Association for Thoracic Surgery doi:10.1016/j.jtcvs.2008.05.040 surgical treatment; however, there have been improvements in perioperative care in both surgery and anesthesia. The development of effective surgical glues and pharmacologic therapies, such as recombinant activated factor $\mathrm{VIIa}^{12,13}$ and aprotinin, ${ }^{14}$ in concert with thromboelastographically targeted therapy have reduced bleeding complications. A greater understanding of the potential for malperfusion has led to the application of a range of cannulation and perfusion techniques, most recently with the adjuncts of antegrade cerebral perfusion and axillary artery cannulation. ${ }^{15,16}$ Newer anesthetic techniques have included using perioperative transesophageal echography, monitoring perfusion and aortic valve function, and managing deep hypothermic circulatory arrest and pharmacologic adjuncts to neuroprotection. ${ }^{9}$ There would therefore seem to be ample reason to believe that the surgical treatment of patients with type A dissection has improved.

The principal purpose of this observational study was to assess whether apparent advances in the management of patients undergoing surgery for type A aortic dissections has translated into better early and mid-term clinical outcomes.

\section{MATERIALS AND METHODS Patient Selection}

All patients undergoing surgical treatment for type A aortic dissection during the period from March of 1992 to March of 2006 at the Bristol Heart Institute were included. To assess and compare the influence of recent trends, we divided patients into 2 equal time frames: before April 1, 1999 (study period A), and April 1, 1999, onward (study period B).

\section{Data Collection and Definitions}

Demographics, preoperative, perioperative, and postoperative data for procedures before April of 1996 were abstracted from the operation notes. 


\section{Abbreviation and Acronym \\ $\mathrm{CT}=$ computed tomography}

Because April of 1996 data were collected prospectively on all patients undergoing cardiac surgery and entered into a database (Patient Analysis and Tracking System (Dendrite Clinical Systems Inc, London, UK), deaths after hospital discharge were identified from mortality data provided by the National Health Service Strategic Tracing Service. All patients were successfully matched to the National Health Service Strategic Tracing Service database.

Arch replacement was defined as operations requiring 2 or more distal anastomoses, one to the distal aorta and one to 1 or more aortic arch branches. Thus, if the undersurface of the aortic arch was replaced (hemiarch) with a single distal anastomosis, it was considered to be an ascending aortic operation only. Renal failure for the purpose of our study was defined as renal impairment requiring hemofiltration. Definitions with respect to the operative priority, premorbid conditions, and postoperative complications are those defined by the National Adult Cardiac Surgical Database and accepted by the Society of Cardiothoracic Surgeons of Great Britain and Ireland (available at www.scts.org).

\section{Surgical Techniques}

The mainstay of surgical treatment was interposition graft replacement of the ascending aorta with valve conservation where possible, but composite root replacement was performed for connective tissue disorders or patients with dilatation or extensive dissection within the sinuses of Valsalva.

During the study period, a range of approaches and developments occurred continually, often without being adopted at a single time point. With respect to the surgical techniques in the second time period, more effective biological glues (Bioglue; Cryolife Europa Ltd, Guildford, UK) replaced the Gelatin-resorcin-formalin (GRF), and aggressive resection of the primary intimal tear with the use of an open distal anastomotic technique has become routine. The tear in the ascending aorta was replaced to the level of the innominate artery. Spiral tears extending along the undersurface of the arch were treated with a beveled distal anastomosis (hemiarch replacements). In time period B, more resections were performed with a hemiarch replacement. Complete aortic arch replacement was performed only for tears located within the aortic arch.

From a perfusion perspective, routine femoral artery cannulation throughout was superseded by the adoption of antegrade reperfusion on completion of the anastomosis via the side arm of the Ante-Flo (Gelweave; Vascutek Ltd, Ann Arbor, Mich) graft conduit, and more recently axillary artery cannulation has been used. Myocardial protection has evolved from cold crystalloid cardioplegia in the first period to routine use of antegrade and retrograde cold blood cardioplegia in the second half of the study.

Additional cerebral protection during deep hypothermic circulatory arrest has changed from widespread use of retrograde cerebral perfusion to circulatory arrest alone to the use of antegrade cerebral perfusion via the axillary artery or endoluminally.

With respect to anesthetic techniques, intraoperative transesophageal echocardiography has been routine in the second period. Bispectral index, which is a monitor of anesthetic depth and incorporates time-domain, frequency-domain, and bispectral analysis of the electroencephalograph, was also used in the second half of the study. Aprotinin was used extensively throughout the study period, and cell savers were used more commonly latterly. Only toward the end of study period B was recombinant factor VIIa used to achieve hemostasis in problematic cases. Thromboelastography has been used exclusively in the latter half (study period B) to guide appropriate use of other blood products.

\section{Statistical Analysis}

Baseline and operative characteristics were compared using the chisquare or Fisher's exact test (categoric variables) or the Wilcoxon ranksum test (continuous variables).

Because the number of adverse outcomes after surgery was few, the results were compared without adjustment for baseline and operative characteristics. Binary variables were compared using Fisher's exact test, and continuous "time to event" variables were compared using the log-rank test. Survival curves were calculated using the Kaplan-Meier method. All analyses were carried out using Stata version 9.2 (Stata Corporation, College Station, Tex).

\section{RESULTS \\ Cohort}

A total of 165 eligible patients were identified. Seventyfour dissections were carried out in the study period A and 91 in study period B. The incidence of referral of type A aortic dissections has remained fairly constant during the last 15 years. Eleven surgeons carried out the operations. The number of procedures ranged from 1 to 61 .

\section{Baseline Characteristics}

Baseline characteristics were similar across the 2 groups (Table 1). The only significant difference was in the percentage of patients with good, moderate, and poor left ventricular function; proportionally fewer patients had good function in the recent era. There were fewer patients with Marfan syndrome in the recent era.

In both periods, the cases were predominantly acute aortic dissections. Only 12 patients $(7.2 \%)$ in total presented with an aortic dissection more than 2 weeks old: $3(4 \%)$ in the early period and $9(10 \%)$ in the more recent era $(P=.15)$. The incidence of patients presenting with preoperative compromise in the 2 eras was also similar (Table 1).

\section{Operative Details}

Details of the surgical procedure are summarized in Table 2. Similar numbers of patients had composite root replacements and interposition grafts in the 2 eras, although in the later period there have been significantly fewer arch replacements. Cardiopulmonary bypass times have increased and circulatory arrest times have decreased over time. The duration of the circulatory arrest time was significantly shorter in the second study period (B).

\section{In-hospital Outcomes}

There were 30 in-hospital deaths $(18.2 \%)$, and the death rate was similar in the 2 time periods $(P=.85$, Table 3$)$. The main causes of early death were cardiac failure, multisystem failure, neurologic causes, bleeding, and gut malperfusion, and the distribution of causes of death in the 2 time periods was similar $(P=.99$, Table 4$)$. Of the in-hospital outcomes examined, the reoperation rates for bleeding, incidence of neurologic, and renal complications, and length of intensive therapy unit stay were similar. The postoperative 
TABLE 1. Baseline characteristics

\begin{tabular}{|c|c|c|c|c|c|}
\hline & \multicolumn{2}{|c|}{$($ Study period A) $(n=74)$} & \multicolumn{2}{|c|}{$($ Study period B) $(\mathbf{n}=91)$} & \multirow[b]{2}{*}{$P$ value $^{\mathrm{a}}$} \\
\hline & $\mathbf{n}$ & $\%$ & $\mathbf{n}$ & $\%$ & \\
\hline Age (y) & $59(45-68)$ & & $62(51-68)$ & & .18 \\
\hline Male patient & 55 & 74 & 63 & 70 & .47 \\
\hline Parsonnet score & $25(20-30)$ & & $28(23-32)$ & & .16 \\
\hline euroScore $^{\mathrm{a}}$ & & & $10(8-12)$ & & \\
\hline Marfan syndrome & 11 & 15 & 6 & 7 & .082 \\
\hline Hypertension & 41 & 55 & 48 & 54 & .85 \\
\hline Peripheral vascular disease & 7 & 9 & 7 & 8 & .69 \\
\hline LV function Good & 61 & 82 & 50 & 60 & $<.001$ \\
\hline Moderate & 13 & 18 & 35 & 39 & \\
\hline Poor & 0 & 0 & 5 & 6 & \\
\hline Preoperative angiogram & 15 & 21 & 9 & 10 & .06 \\
\hline Cardiac arrest & 1 & 1.3 & 3 & 3.2 & .63 \\
\hline Renal failure & 2 & 2.7 & 4 & 4.3 & .69 \\
\hline Neurologic event & 3 & 4 & 2 & 2.1 & .73 \\
\hline Mesenteric ischemia & 1 & 1.3 & 3 & 3.2 & .63 \\
\hline Limb ischemia & 5 & 6.7 & 4 & 4.3 & .52 \\
\hline SVC obstruction & 1 & 1.3 & & & \\
\hline
\end{tabular}

$\overline{S V C}$, Superior vena cava. ${ }^{\mathrm{a}} \mathrm{Not}$ recorded in first era.

length of hospital stay has increased over time, from a median of 13 days (interquartile range 10-16 days) for operations before April of 1999 to a median of 15 days (interquartile range 10-24 days) operation since then $(P=.034)$.

Of the 16 patients who had a permanent stroke, 5 subsequently died in the hospital. Four of 26 patients who developed renal failure died (multisystem failure in 3 and associated gut malperfusion in 1).

\section{Mid-term Outcome}

The median follow-up of survivors who underwent before April of 1999 (time period A) was 7.9 years. The survival at 3 years, 5 years, and 7 years in this group was $71.5 \% .70 .1 \%$, and $61.2 \%$, respectively. However, because the follow-up was shorter in the later period (time period B), the follow-up was censored at 3 years in each group for comparison. There were 44 deaths in total over the 3 years; $32 \%$ occurred within 1 day of surgery. Overall, 3-year survival has not changed significantly over time (log-rank test, $P=.50$, Figure 1 ).

\section{DISCUSSION}

In this study we have been unable to demonstrate any improvement in early and mid-term clinical outcome for patients undergoing surgery for type A dissection at the Bristol Heart Institute during a 15-year period. To our knowledge, this series represents the largest consecutive institutional experience from a UK center.

Taken as a whole, these outcomes are in the middle of contemporary published results. ${ }^{2-4}$ They do not match the best results published, ${ }^{8,9}$ although caution has been advised

TABLE 2. Operative characteristics

\begin{tabular}{|c|c|c|c|c|c|}
\hline & \multicolumn{2}{|c|}{$($ Study period A) $(n=74)$} & \multicolumn{2}{|c|}{$($ Study period B $)(n=91)$} & \multirow[b]{2}{*}{$P$ value $^{\text {a }}$} \\
\hline & $\mathbf{N}$ & $\%$ & $\mathbf{N}$ & $\%$ & \\
\hline Arch replacement & 12 & 16 & 4 & 4 & .011 \\
\hline Composite root replacement & 21 & 28 & 30 & 33 & .53 \\
\hline Interposition graft & 49 & 66 & 62 & 68 & .79 \\
\hline Without aortic valve replacement & 29 & 59 & 43 & 69 & .52 \\
\hline With aortic valve replacement & 6 & 13 & 6 & 10 & \\
\hline Redo procedure & 6 & 8 & 11 & 12 & .39 \\
\hline Circulatory arrest & 59 & $80 \%$ & 79 & $87 \%$ & .22 \\
\hline Circulatory arrest time (min) & \multicolumn{2}{|c|}{$37.5(31-45)$} & \multicolumn{2}{|c|}{$31(26.5-39)$} & .0095 \\
\hline Bypass time (min) & \multicolumn{2}{|c|}{$149(118-200)$} & \multicolumn{2}{|c|}{$166(141-202)$} & .047 \\
\hline Cross clamp time (min) & \multicolumn{2}{|c|}{$80(54-100)$} & \multicolumn{2}{|c|}{$80(63-111)$} & .30 \\
\hline Mitral valve surgery & 1 & 1 & 2 & 2 & .58 \\
\hline CABG & 9 & 12 & 17 & 19 & .25 \\
\hline No. of grafts ${ }^{\mathrm{a}}$ & 1.2 & 0.4 & 1.5 & 0.6 & .20 \\
\hline
\end{tabular}

$C A B G$, Coronary artery bypass graft. ${ }^{\mathrm{a}} \mathrm{Grafted}$ patients only (mean and standard deviation). 
TABLE 3. Outcomes after surgery

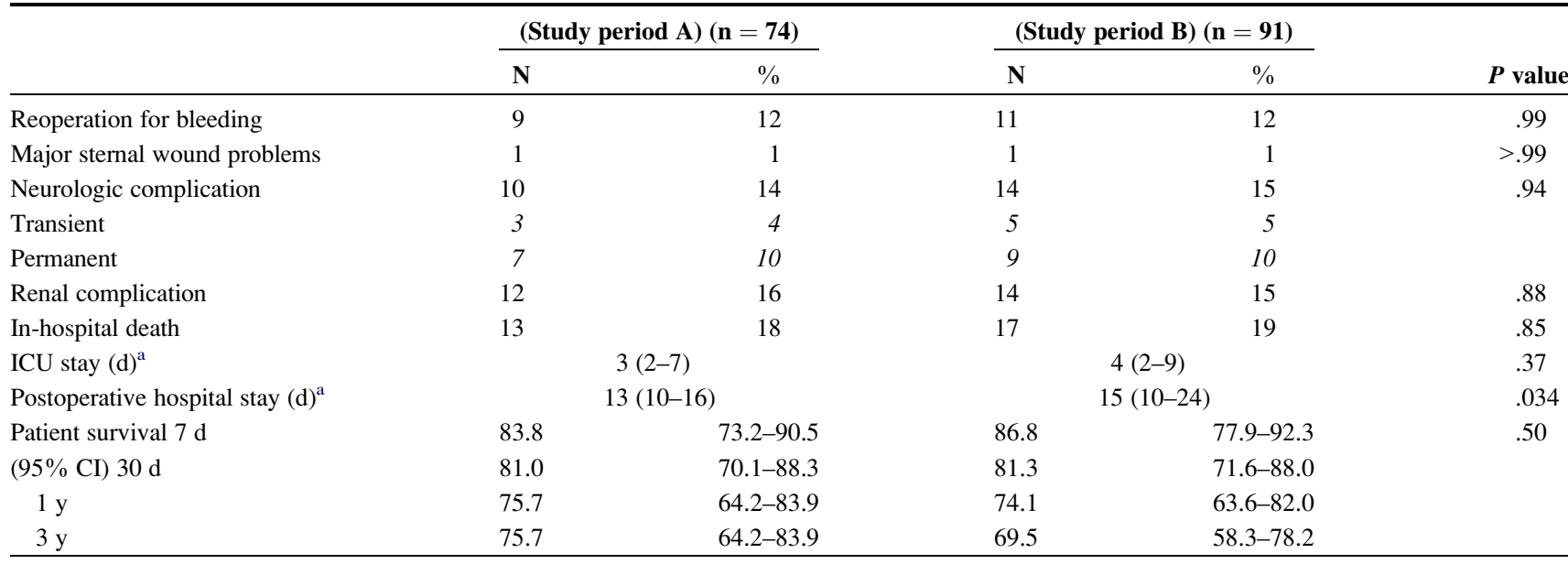

$I C U$, Intensive care unit; $C I$, confidence interval. ${ }^{\mathrm{a}}$ Median and interquartile range.

against believing that limited single surgeon series with low mortalities provide an accurate reflection of the likely potential outcome for a patient with type A dissection. ${ }^{17}$ Studies collecting outcome from large numbers of patients as registry data probably reflect more accurately potential outcome. The International Registry of Aortic Dissections ${ }^{3}$ and the Swedish ${ }^{4}$ and Taiwanese ${ }^{18}$ registries report early mortalities of $25.1 \%, 22.4 \%$, and $19.6 \%$, respectively, in type A aortic dissection. The mid-term survival of the patients in either era of our study period was similar to the results published by some higher volume centers. ${ }^{2,10}$

A significant number of studies have analyzed outcome for type A aortic dissection and typically have identified factors relating to the patient and the mode of presentation as the primary determinants of outcome. Patients with previous cardiac surgery, shock with tamponade, preoperative evidence of stroke, and coronary, visceral, or peripheral malperfusion tend to have a worse outcome..$^{2,3,6,9-11}$ Despite these considerations, in a single institution, with a considerable evolution in techniques it is reasonable to expect an improvement in outcome.

At the Bristol Heart Institute, the senior surgical personnel have been relatively constant during this time period. Although the idea of a master surgeon achieving excellence through undertaking high volumes of such surgery is an attractive one, this is an unproven hypothesis and an impractical proposition. In the area of thoracic aortic surgery, we have previously presented data suggesting that a group of surgeons adopting similar techniques can produce equivalent results. ${ }^{19}$ Given that type A dissection presents as an emergency and requires prompt intervention, service is best provided by a group of surgeons with the necessary expertise, and we favor strongly the approach of Bavaria and associates $^{8}$ of an agreed institutional protocol.

At the Bristol Heart Institute, we have identified changes in several areas in the treatment of these patients during the 15-year period, in terms of the surgical approach, perfusion and blood conservation techniques, anesthetic neuroprotective strategies, and routine use of transesophageal echocardiography. Although these changes have been a continuum, there are major differences between the 2 study periods 1992 to 1999 and 1999 to 2007 in our conduct of this surgery. The observation that major clinical outcomes have not improved is rather disappointing. Both the Houston ${ }^{5}$ and Cleveland Clinic ${ }^{6}$ groups have shown better outcomes with more recent operation dates. However, both of these studies cover a much longer time scale than our own. In contrast, other authors have indicated that although techniques have evolved, mortality has not improved in recent years. ${ }^{2}$ Olsson and associates, ${ }^{4}$ reporting data from the Swedish

TABLE 4. Causes of death

\begin{tabular}{|c|c|c|c|c|}
\hline & \multicolumn{2}{|c|}{$\underline{\text { Study period A }(\text { No. of deaths }=13)}$} & \multicolumn{2}{|c|}{ Study period B $($ No. of deaths $=17)$} \\
\hline & $\mathbf{n}$ & $\%$ & $\mathbf{n}$ & $\%$ \\
\hline Multisystem failure & 3 & 23 & 3 & 18 \\
\hline Cardiac failure & 4 & 31 & 7 & 40 \\
\hline Neurologic event & 3 & 23 & 2 & 12 \\
\hline Bleeding & 2 & 16 & 3 & 18 \\
\hline Visceral malperfusion & 1 & 7 & 1 & 6 \\
\hline Other & & & 1 & 6 \\
\hline
\end{tabular}




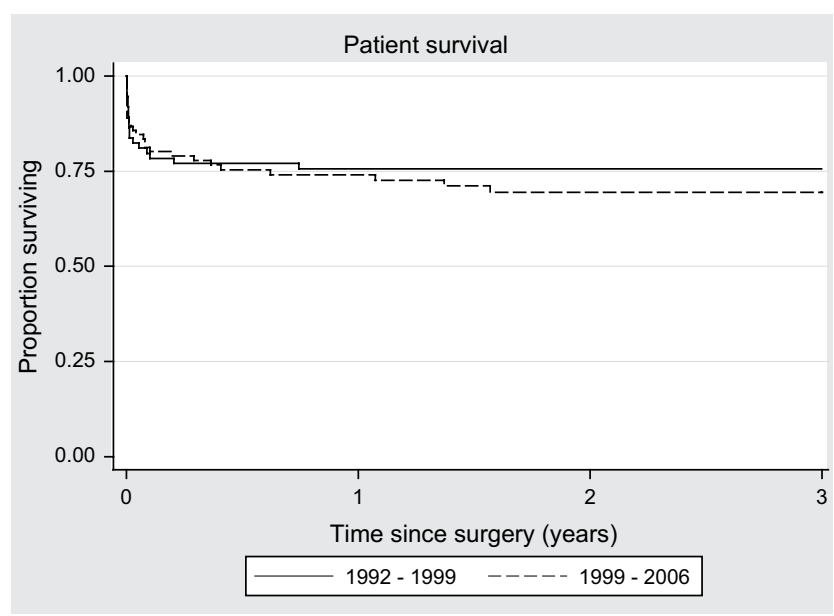

FIGURE 1. Kaplan-Meier survival estimates by era.

registry, have recorded improved outcomes for patients requiring aneurysm resection, whereas those for aortic dissection have not shown a similar improvement.

Bachet ${ }^{17}$ recently commented on this issue and concluded that although we must congratulate our surgical colleagues who present the best results in observational series, we must also recognize the likely regression toward the mean effect of larger series. We must also acknowledge the catastrophic nature of the disease, its acute and devastating natural history, and the end-organ ischemia that results from cerebral, visceral, or coronary malperfusion. Despite improved surgical expertise, this may continue to remain a challenge to further improve the outcomes.

In a 15-year experience of 165 consecutive patients with acute type A dissection treated at a single institution, an in-hospital mortality of $18.2 \%$ was achieved, similar to that observed in large series reported in the literature. Although we must continue to strive to achieve the best outcomes, we should not forget the nature and devastating presentation of type A aortic dissections.

In the future, further development of radiologic techniques, such as the 64-slice CT coronary angiography and integrated single-photon emission CT and CT coronary angiography, ${ }^{20}$ may provide surgeons anatomic information about the presence of coronary artery disease. A greater effort to diagnose coronary artery disease using noninvasive means may reduce the need for salvage coronary artery bypass grafting, which is associated with a significantly higher mortality, and this is likely to be particularly so in an aging population. ${ }^{21}$ Efforts should perhaps also be aimed at primary prevention of the condition by more aggressive management of risk factors and by identifying and following patients at risk of an aortic dissection.

\section{Limitations}

There were 2 important limitations of our study. First, acute aortic dissections at the Bristol Heart Institute are managed by a group of surgeons with a range of clinical experience and techniques. Although there is a broad consensus over surgical practice, there were individual variations. For instance, axillary artery cannulation and antegrade cerebral perfusion was adopted in 2001 at the Bristol Heart Institute. However, adoption of this technique was surgeon dependent and the overall number of patients in whom this technique was used was low. This may in part explain why antegrade cerebral perfusion failed to demonstrate a benefit on neurologic outcomes. A further period of observation may be necessary because these techniques have now been more widely disseminated to properly evaluate their impact on outcome.

The second important limitation relates to the study size. Increased comorbidity and concomitant procedures in the more recent era may have counterbalanced any improvement in survival, and perhaps a multivariate analysis would be of interest. However, only 30 in-hospital deaths in this series may mean the data are insufficient to allow any meaningful conclusions to be drawn from such an analysis.

\section{References}

1. Green RG, Kron IL. Aortic dissection. In: Cohn HL, Edmunds HL Jr., eds. Cardiac Surgery in the Adult. New York: McGraw Hill Companies; 2003: 1097.

2. Ehrlich MP, Ergin MA, McCullough JN, Lansman SL, Galla JD, Bodian CA, et al. Results of immediate surgical treatment of all acute Type A dissections. Circulation. 2000;102(suppl III):248-52.

3. Trimarchi S, Nienaber CA, Rampoldi V, Myrmel T, Suzuki T, Mehat RH, et al. Contemporary results of surgery in acute type A aortic dissection: The International Registry of Acute Aortic Dissection experience. J Thorac Cardiovasc Surg. 2005;129:112-22.

4. Olsson C, Eriksson N, Stahle E, Thelin S. Surgical and long-term mortality in 2634 consecutive patients operated on the proximal thoracic aorta. Eur J Cardiothorac Surg. 2007;31:963-9.

5. Svensson LG, Crawford ES, Hess KR, Coselli JS, Safi HJ. Dissection of the aorta and dissecting aortic aneurysms. Improving early and long term results. Circulation. 1990;82(Suppl IV):24-8.

6. Sabik JF, Lytle BW, Blackstone EH, McCarthy PM, Loop FD, Cosgrove DM. Long-term effectiveness of operations for ascending aortic dissections. $J$ Thorac Cardiovasc Surg. 2000;119:946-62.

7. Mesh T, Dossche KME, Morshuis WJ, Kelder JC, Waanders FGJ, Schepens MAAM. Is extended arch replacement for acute type A aortic dissection an additional risk factor for mortality? Ann Thorac Surg. 2003;76:1209-14.

8. Westaby S, Saito S, Katsumata T. Acute type A dissection: conservative methods provide consistently low mortality. Ann Thorac Surg. 2002;73:707-13.

9. Bavaria JE, Brinster DR, Gorman RC, Woo YJ, Gleason T, Pochettino A. Advances in the treatment of acute type A dissection: an integrated approach. Ann Thorac Surg. 2002;74:S1848-52.

10. Kazui T, Washiyama N, Bashar AHM, Terada H, Suzuki T, Ohkura K, et al. Surgical outcome of acute type A aortic dissection: analysis of risk factors. Ann Thorac Surg. 2002;74:75-82.

11. Long SM, Tribble GC, Raymond DP, Fiser SM, Kaza AK, Kern JA, et al. Preoperative shock determines outcome for acute type A aortic dissection. Ann Thorac Surg. 2003;75:520-4.

12. Tritapepe L, Santis VD, Vitale D, Nencini C, Pellegrini F, Landoni GL. Recombinant activated factor VII for refractory bleeding after acute aortic dissection surgery: a propensity score analysis. Crit Care Med. 2007;35:1685-90.

13. Raivio P, Suojaranta-Ylinen R, Kuitunen AH. Recombinant factor VIIa in the treatment of postoperative haemorrhage after cardiac surgery. Ann Thorac Surg. 2005;80:66-71

14. Sedrakayan A, Wu A, Sedrakyan G, DienerWest M, Tanquilli M, Elefteriades J. Aprotinin use in thoracic aortic surgery; safety and outcomes. $J$ Thorac Cardiovasc Surg. 2006;132:909-17. 
15. Pasic M, Schubel J, Bauer M, Yankah C, Kuppe H, Weng YG, et al. Cannulation of the right axillary for surgery of acute type A aortic dissection. Eur J Cardiothorac Surg. 2003;24:231-6.

16. Moizumi Y, Motoyoshi N, Sakuma K, Yoshida S. Axillary artery cannulation improves operative results for acute type A aortic dissection. Ann Thorac Surg. 2005; 80:77-83.

17. Bachet J. Acute type A aortic dissection: can we dramatically reduce the surgical mortality. Ann Thorac Surg. 2002;73:701-3.

18. Yu HY, Chen YS, Huang SC, Lin FY. Late outcome of patients with aortic dissection: study of a national database. Eur J Cardiothorac Surg. 2004;25:683-90.
19. Narayan P, Caputo M, Rogers C, Angelini G, Bryan AJ. Early and mid-term outcomes of surgery of the ascending aorta/arch: is there a relationship with caseload? Eur J Cardiothorac Surg. 2004;25:676-82.

20. Rispler S, Keidar Z, Ghersin E, Roguin A, Soil A, Dragu R, et al. Integrated single-photon emission computed tomography and computed tomography coronary angiography for the assessment of hemodynamically significant coronary artery lesions. J Am Coll Cardiol. 2007;50:1469-75.

21. Narayan P, Caputo M, Rogers C, Angelini G, Bryan AJ. Influence of concomitant coronary artery bypass graft on outcome of surgery of the ascending aorta/arch. Heart. 2007;93:232-7. 